\section{The Profession and the Big Picture}

Neville Holmes, University of Tasmania ilar, if not worse, elevation in temperature is probable by the end of this century. This strongly implies that human society faces a catastrophe. Yet this impending disaster is still not widely realized. As professionals, we have a duty to spread this information.

The differences between today's climate changes and those of past catastrophes derive from their source: Human activity largely drives today's change, primarily through the extraction of huge amounts of carbon-as gas, oil, and coal-from underground. When used, these minerals subse-

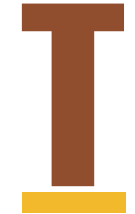

his column has on occasion emphasized that the responsibilities of computing professionals-indeed of any learned profession's members-go beyond acquiring and applying technical experience and wisdom and beyond acting in their clients' best interests. The greatest professional responsibility is to act in the best interests of the society to which the profession owes its status.

By definition, human society's greatest interest is its continued existence. It seems the greatest threat to that continuance-climate change-is at last being given credence beyond scientific and environmental circles. The computing profession will play a crucial role in the battle to mitigate the effects of climate change and, if possible, adapt to them.

Therefore, we now share a responsibility to inform ourselves of the facts and press for the strategies and measures that we believe will be most effective in combating this threat.

\section{CLIMATE CHANGE}

The term climate refers to both the pattern of weather over a typical year and variations from typicality over longer periods. Climate varies from place to place, determining the kind of plants and wildlife that can live in a given location and, to a lesser degree, the kind and quality of life people can have there.

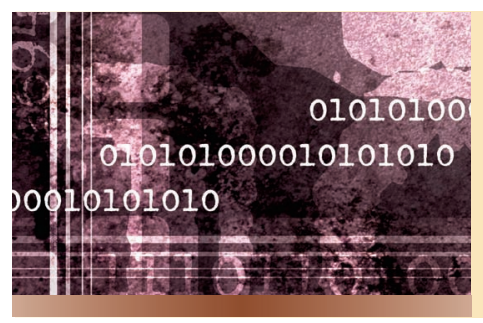

The profession must tackle social challenges, the biggest of which is human society's continued survival.

Geological records show quite clearly that climate can change greatly over various time scales. Historically, meteorological records show that the global climate has been gradually warming over the past century, while more recent meteorological records show that extremes of weather are becoming more frequent.

Although these meteorological records build a picture of anthropogenic climate change validated by scientific modeling and accepted by practicing climate scientists (www.sciencemag.org/ cgi/content/full/306/5702/1686), some people still deny the facts. As professionals avowing rationality, we should become familiar with these facts and be ready to promptly counter such denials. The most popular denial at the moment is Michael Crichton's deceptive State of Fear, authoritively rebutted at www. realclimate.org.

Geological records show that many of the catastrophic discontinuities that separate major eras coincide with elevated temperatures that result from atmospheric changes. Simple projections of current trends show that a sim- quently convert to atmospheric carbon dioxide, $\mathrm{CO}_{2}$, presently at double the rate at which the Earth can absorb it.

This $\mathrm{CO}_{2}$ buildup has been accepted at an international level as harmful and man-made, although the acceptance lacks any sense of emergency. Indeed, critics typically advance arguments against doing anything about it because a discounted cash flow analysis suggests that countermeasures would be cheaper if postponed.

An engineer could easily demolish this absurd kind of argument, which is based on a superficial estimate of the costs involved and an assumption that climate has a simple linear behavior. Like all complex systems, Earth has time constants that could well induce dramatically nonlinear behavior, even unto catastrophe, that no delayed action could avoid.

\section{PREDICTION}

Human society must react to climate change. Just how it should react depends on reliable predictions of what will happen and how quickly, Continued on page 102 
and what effect various countermeasures will have. Such predictions will rely heavily on digital technology. The quality of a digital climate model depends on two factors: the data available and the model itself.

Having large amounts of the right kind of data available ensures that the simulation is more reliable and allows refining the model by comparing predictions to outcomes. Digital technology could and should be more widely used to gather, store, and distribute meteorological and geological data.

Global climate models are already tremendously complex, and their development makes them steadily more so. Such models are based on 3D spatial grids, so halving the grid spacing to improve accuracy requires eight times as many data points. Refining the model, as discrepancies between prediction and outcome are explained, leads to the need for more data and computation at each grid point.

Presently, there are two approaches to climate simulation: distributed, as practiced at www.climateprediction. com, and using supercomputers. Both approaches must be improved continually.

This raises the basic problem that prediction cannot simply be a matter of projection. Too many contingencies also need scientific study and modeling: natural contingencies such as methane burps and ocean current changes and human contingencies such as mass migration resulting from the imminent Peruvian parching and the eventual Bangladeshi submersion. As researchers develop a better understanding of these contingencies, they must build the likely effects, singly and in combination, into the overall climate model.

From my reading, I suspect that these computations will require special-purpose multiprocessors with, for example, one processor per grid point. The arithmetic might also need improvement to lessen the accumulation of error in such large calculations. Whatever the case, the computing pro- fession will play a crucial role in developing climate modeling.

\section{MITIGATION}

The international community already recognizes that the human activity causing global warming must be curbed. The Kyoto Agreement (http://unfccc. int/ essential_background/kyoto_protocol/ items/2830.php) intended just that but seems unlikely to have any nonpolitical effect.

\section{We need to slow the accelerating addition of net $\mathrm{CO}_{2}$ to the atmosphere.}

The problem seems to be that key political agents either do not know or do not accept the relevant facts. For example, at the recent two-week UN conference on climate change in Buenos Aires, the US representatives reportedly said that their government wants to concentrate on long-term programs to develop cleaner-burning energy technologies (www.state.gov/g/ oes/rls/fs/2004/38641.htm). This statement implies that they do not understand that dirty burning has been lessening global warming, while the burning of fossil fuels adds $\mathrm{CO}_{2}$ to the atmosphere and-perhaps just as seriously in the long run-any burning removes oxygen from it.

There is no doubt that we need to slow the accelerating addition of net $\mathrm{CO}_{2}$ to the atmosphere, even though it is not the only cause of climate change. Indeed it's possible that soon we will need to actually reduce the $\mathrm{CO}_{2}$ content to avoid catastrophe. Better modeling would give us a better idea of what's needed and provide a more persuasive argument to make to the politicians.

Because the situation calls for political action, informing the public of the relevant facts and projections might seem a practical activity, one in which computing professionals could use the Web and media as useful conduits. However, this assumes that an effec- tive percentage of the public can understand the relevant scientific evidence and reasoning.

This assumption might well be wrong. For example, The Nation's Report Card: Mathematics 2000 noted that more than one-third of US high school seniors lack basic proficiency in mathematics (http://nces.ed.gov/ nationsreportcard/pdf/main2000/2001 517.pdf). Worse, fewer than one-sixth have better than a basic proficiency.

Clearly, the computing profession should be pushing for the use of computers in schools simply to inculcate such basic skills, not just to ensure that younger people can understand what the climate has in store for them.

If we accept the need for practical and intense mitigation, a boost to education is crucial because of the underlying need for more scientists to analyze and model the climate and for more engineers to design and implement the machinery for mitigation. Further, given digital technology's potential to help educators, scientists, and engineers be more effective, more computing professionals will be needed. And their education must focus on the problems they will face.

\section{ADAPTATION}

Engineers in general, and computing professionals in particular, understand professionally the likely short-term and long-term behavior of complex systems. The Earth's climate is changing now, will change dramatically within a few human generations, and could change catastrophically in the longer term. These changes will drastically affect human society. If this is not soon accepted globally and officially, the world's scientists and engineers must take a large part of the blame.

The greatest danger is that human society will not adapt to the inevitable changes. Both mitigation and adaptation must be technologically based, just as the climate change itself is. In the worst case, the human effects of widespread starvation and thirst brought on by glacier disappearance alone 
could cause social disruption widespread enough to block the development and use of mitigation technology.

Governments will need to use technology of many kinds, necessarily supported by digital technology, in critically threatened areas in the immediate future simply to keep people there supplied with food and water.

In the medium term, agriculture as we know it might not survive if we cannot stop the spread of deserts. Should this occur, scientists would need to develop ways to industrially manufacture food. The flooding of low-lying coastal areas will require either constructing enormous levee banks or relocating many of the world's largest cities and densest rural populations. Increasing heat will mean that a large proportion of the world's population will depend on air conditioning for its very survival—even now, thousands die of extreme summer heat each year. Extremes of weather will require constructing buildings and the infrastructure more sturdily or even completely redesigning them.

In the long run, if mitigation is unsuccessful, the human race will be forced to live in a completely artificial environment, isolated from Earth's climate. Achieving this will present a huge technological challenge. On the bright side, if we succeed, we should be able to colonize the Moon and Mars as well.

\section{MOTIVATION}

Some readers may view this essay as mere scaremongering. I intend it to scare, but only because my reading has convinced me that the human race faces truly frightening prospects and that we might indeed already be doomed, at least as a civilization.

I ask only that those of you who remain unconvinced of the reality of these threats at least read some of the resources I have found-all directly or indirectly available through our wonderful Web and discoverable using its search engines. George Monbiot's short essay, "Goodbye, Kind World" (www.monbiot.com/archives/2004/08/ 10/goodbye-kind-world-/), shows that I am not alone in my apprehensions. Mark Lynas's book, High Tide (Flamingo, 2004; www.marklynas.org), is a persuasive and well-documented eyewitness account of some climate change effects already being felt.

More details can be found at govern- ment Web sites such as the Intergovernmental Panel on Climate Change (www.ipcc.ch), academic Web sites such as that for the American Institute of Physics (www.aip.org/history/climate/ summary.htm), and activists' Web sites such as www.worldwatch.org and www.climateark.org.

$\mathbf{T}$ echnology has almost entirely shaped the outward aspects of our civilization, and civilization's use of technology will certainly determine its own fate. Given that digital technology has become the main enabler of other technologies, this issue has undeniable relevance to the computing profession. In this case, we face the real danger that inaction by our profession and others might force us to share the fate of the apocryphal frog who, oblivious to his imminent demise, boiled to death in a gradually warming saucepan.

Neville Holmes is an honorary research associate at the University of Tasmania's School of Computing. Contact himatneville.holmes@utas.edu.au. Details of citations in this essay and links to further material are at www. comp.utas.edu.au/users/nholmes/prfsn.

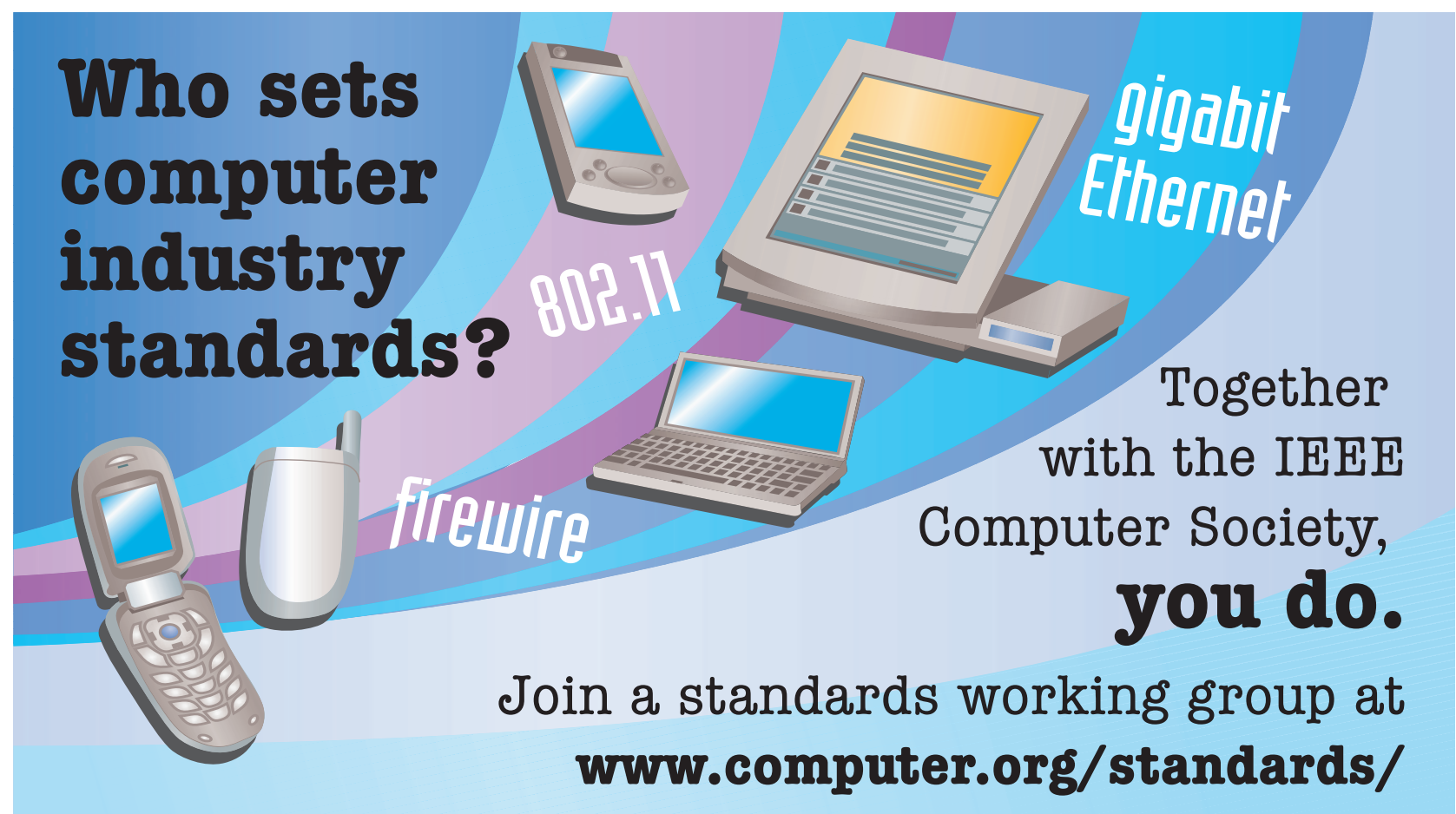

